\title{
Supporting Information for Activation of Oxygen and Hydrogen Peroxide by \\ Copper(II) Coupled with Hydroxylamine for Oxidation of Organic Contaminants
}

Hongshin Lee ${ }^{\dagger, \S}$, Hye-Jin Lee ${ }^{\S}$, Jiwon Seo $^{\S}$, Hyung-Eun Kim ${ }^{\S}$, Yun Kyung Shin ${ }^{\ddagger}$, Jae-Hong Kim ${ }^{\dagger}$, Changha Lee ${ }^{\S, *}$

${ }^{\dagger}$ Department of Chemical and Environmental Engineering, Yale University, New Haven, Connecticut 06511, United States

ॠSoutheast Sea Fisheries Research Center, National Fisheries Research and Development Institute (NFRDI), 397-68 Sanyangilju-ro, Tongyeong-si, Gyeongsangnam-do 53085, Republic of Korea

${ }^{\S}$ School of Urban and Environmental Engineering, and KIST-UNIST-Ulsan Center for Convergent Materials (KUUC), Ulsan National Institute of Science and Technology (UNIST), 50 UNIST-gil, Ulsan 44919, Republic of Korea

*Corresponding author.

Phone: +82-052-217-2812; Fax.: +82-052-217-2809; E-mail: clee@unist.ac.kr

The supporting information includes 13 pages, 12 figures. 
(a)

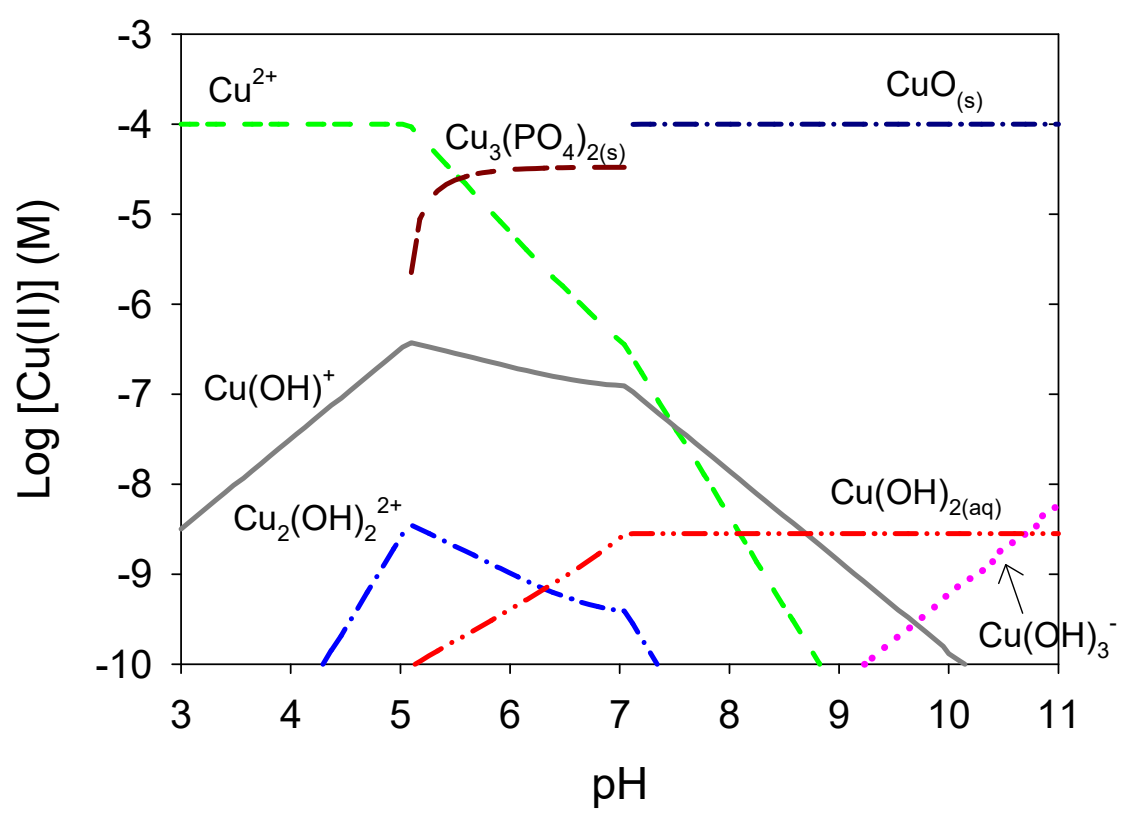

(b)

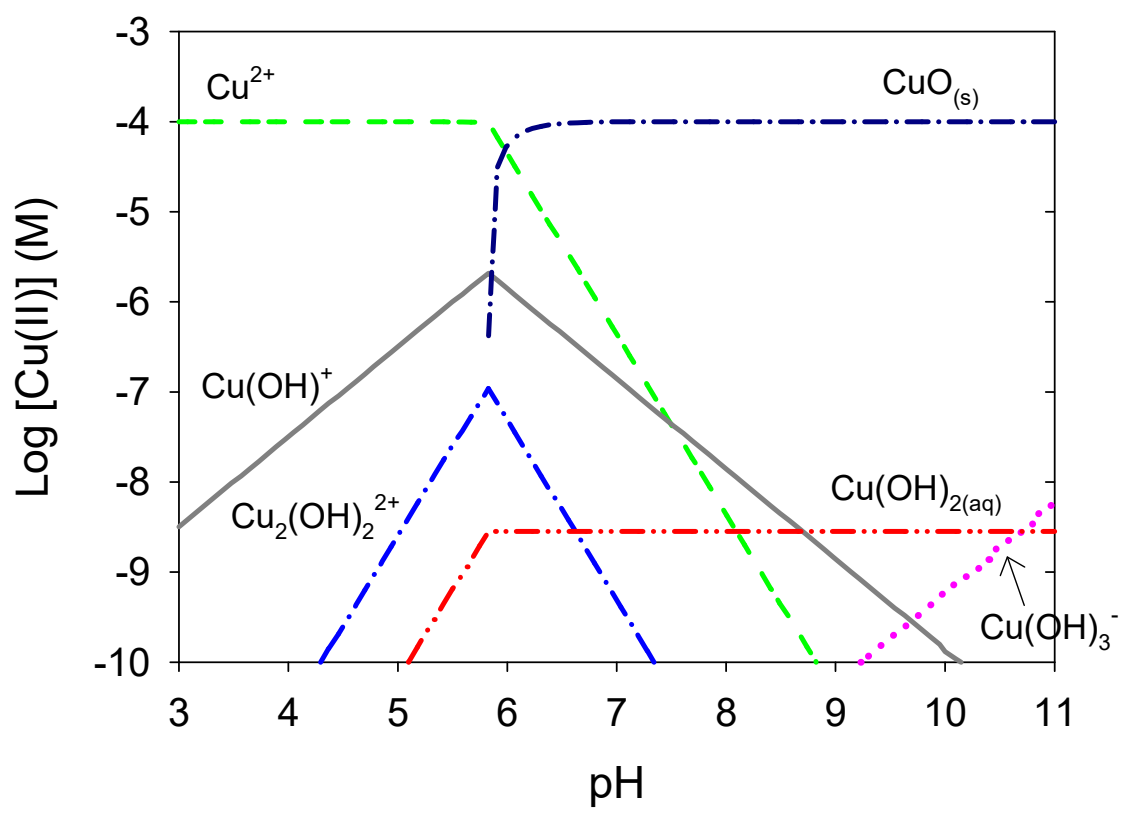

Figure S1. Speciation of $\mathrm{Cu}(\mathrm{II})$, (a) with and (b) without phosphate buffer $(1 \mathrm{mM})\left([\mathrm{Cu}(\mathrm{II})]_{\mathrm{t}}=0.1\right.$ $\mathrm{mM}$; calculated using MINEQL+ with the stability constants provided in the database). 


\section{S1. Analytical procedures of $p H B A$ and other products using the RSLC/orbitrap MS/MS}

system:

The RSLC separation was performed with an acclaim ${ }^{\mathrm{TM}} \mathrm{C} 18$ column $(150 \mathrm{~mm} \times 2.1 \mathrm{~mm}, 2.2 \mu \mathrm{m}$; Thermo Fisher Scientific Inc.) with a mobile phase composed of $0.1 \%$ formic acid solution and acetonitrile at a flow rate of $0.3 \mathrm{~L} \mathrm{~min}^{-1}$. The mass detection was carried out in a negative electrospray ionization (ESI) mode. The operating parameters of the mass spectrometer include: spray voltage $=3.1 \mathrm{kV}$, capillary temperature $=320{ }^{\circ} \mathrm{C}$, maximum injection time $=100 \mathrm{~ms}$, sheath gas flow rate $=40$ arbitrary units, auxiliary gas flow rate $=10$ arbitrary units, $\mathrm{S}$ lens $\mathrm{RF}$ level $=65$, auxiliary gas heater temperature $=300{ }^{\circ} \mathrm{C}$. The mass spectra were acquired in a selected ion monitoring (SIM) mode from 135.52 to $138.52 \mathrm{~m} / \mathrm{z}$ at a resolution of 35000 , an automatic gain control (AGC) target value of $5 \times 10^{4}$, and a maximum injection time of $100 \mathrm{~ms}$. Accurate mass measurements were attained within $5 \mathrm{ppm}$ of the theoretical mass. Only pHBA was quantified using a standard solution (Figure 3c, Figures S8-S10); other products were qualitatively analyzed and their relative abundances (peak areas) were presented (Figure S4). 


\section{S2. Product analysis of $\mathrm{BA}$ oxidation by the $\mathrm{Cu}(\mathrm{II}) / \mathrm{HA}$ system:}

The analysis using the RSLC/orbitrap MS/MS system identified the BA oxidation products including hydroxybenzoates, dihydroxybenzoates, trihydroxybenzoates, nitrobenzene, nitrobenzoates, and nitro-hydroxybenzoates. The pathways of BA oxidation were depicted in Figure S2. Based on the relative abundance of adduct ions, hydroxybenzoates, dihydroxybenzoates, and nitrobenzene (products 1, 2,6) were major oxidation products, and trihydroxybenzoates, nitrobenzoates, and nitro-hydroxybenzoates (products 3, 4, 5) were minor ones (Figures S3 and S4).

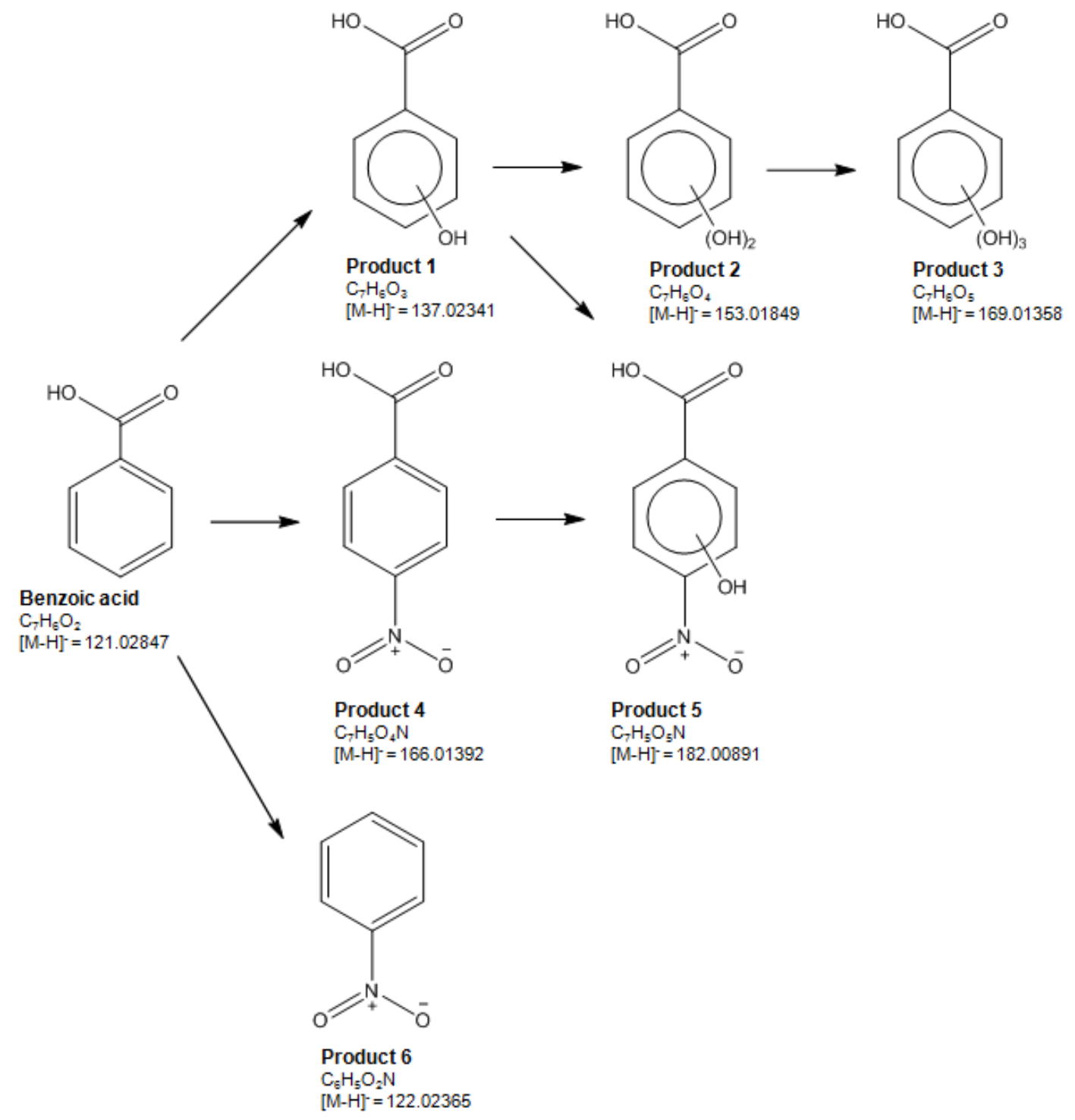

Figure S2. Pathways of BA oxidation by the $\mathrm{Cu}(\mathrm{II}) / \mathrm{HA}$ system. 
(a) Product $1\left(\mathrm{C}_{7} \mathrm{H}_{6} \mathrm{O}_{3},[\mathrm{M}-\mathrm{H}]^{-}=137.02341\right)$

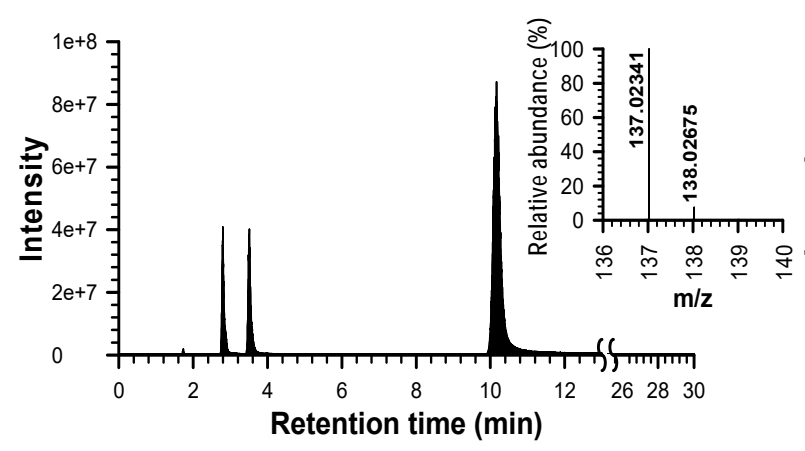

(c) Product $3\left(\mathrm{C}_{7} \mathrm{H}_{6} \mathrm{O}_{5},[\mathrm{M}-\mathrm{H}]^{-}=169.01358\right)$

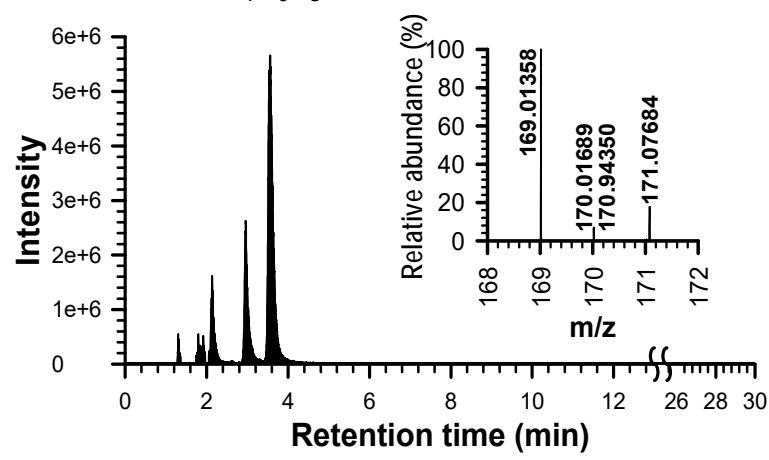

(e) Product $5\left(\mathrm{C}_{7} \mathrm{H}_{5} \mathrm{O}_{5} \mathrm{~N},[\mathrm{M}-\mathrm{H}]^{-}=182.00891\right)$

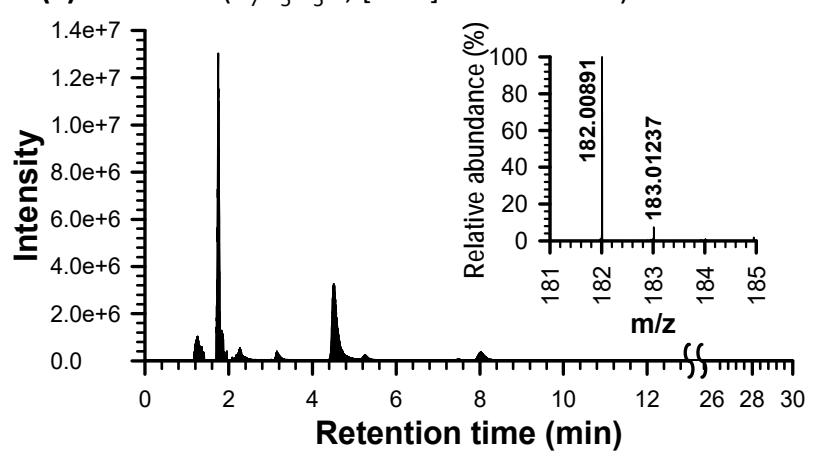

(b) Product $2\left(\mathrm{C}_{7} \mathrm{H}_{6} \mathrm{O}_{4},[\mathrm{M}-\mathrm{H}]^{-}=153.01849\right)$

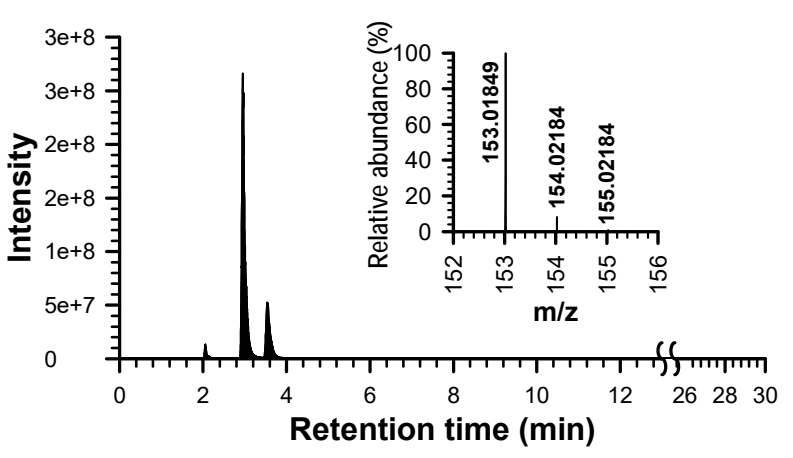

(d) Product $4\left(\mathrm{C}_{7} \mathrm{H}_{5} \mathrm{O}_{4} \mathrm{~N},[\mathrm{M}-\mathrm{H}]^{-}=166.01392\right)$

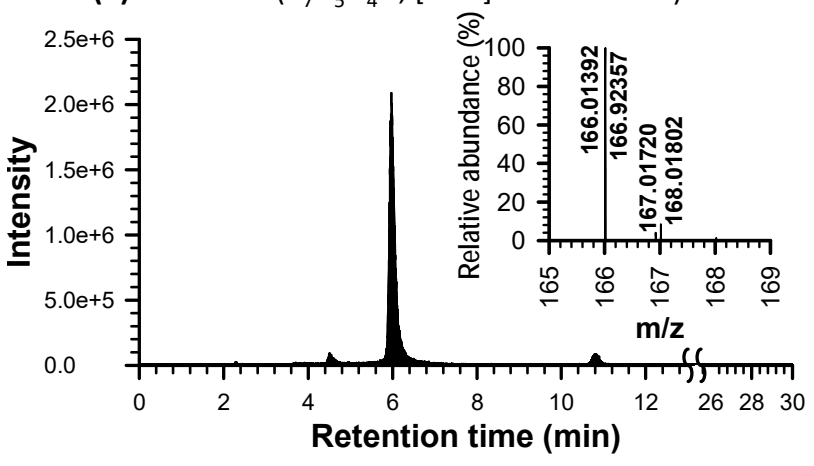

(f) Product $6\left(\mathrm{C}_{6} \mathrm{H}_{5} \mathrm{O}_{2} \mathrm{~N},\left[\mathrm{M}-\mathrm{H}^{-}=122.02365\right)\right.$

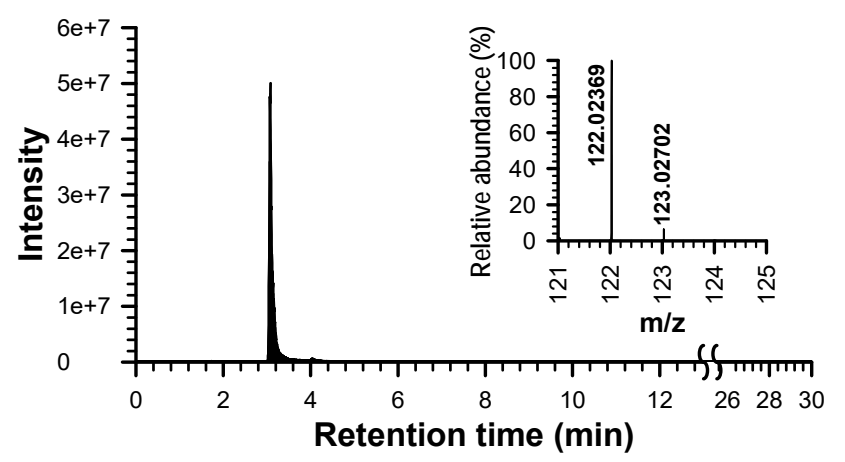

Figure S3. LC/MS spectra of BA oxidation products $\left([\mathrm{BA}]_{0}=0.1 \mathrm{mM},[\mathrm{Cu}(\mathrm{II})]_{0}=0.1 \mathrm{mM},[\mathrm{HA}]_{0}=\right.$ $5 \mathrm{mM}, \mathrm{pH}=7$, reaction time $=120 \mathrm{~min})$. 

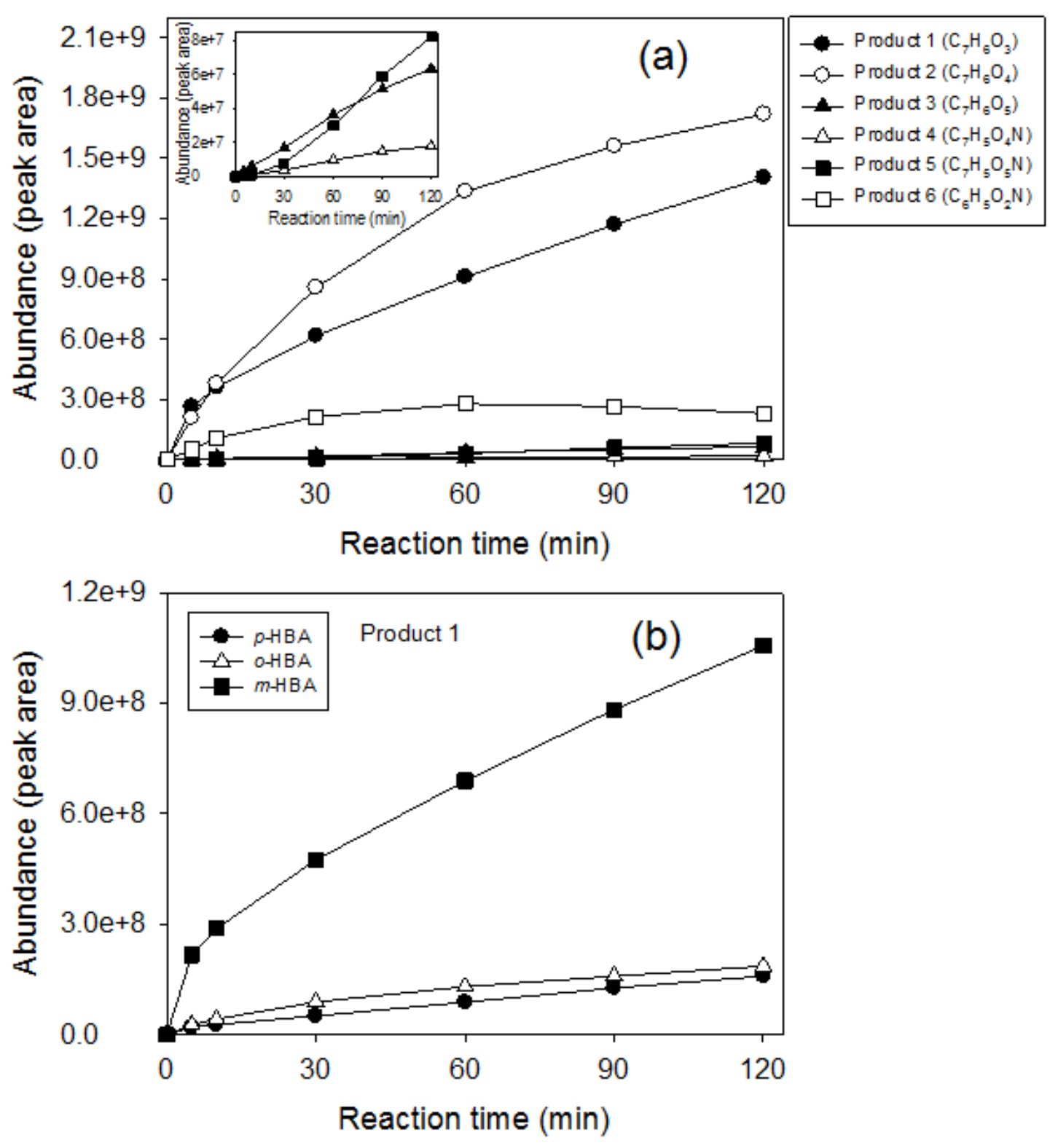

Figure S4. Abundance of products produced by the BA oxidation in the $\mathrm{Cu}(\mathrm{II}) / \mathrm{HA}$ system as a function of reaction time $\left([\mathrm{BA}]_{0}=0.1 \mathrm{mM},[\mathrm{Cu}(\mathrm{II})]_{0}=0.1 \mathrm{mM},[\mathrm{HA}]_{0}=5 \mathrm{mM}, \mathrm{pH}=7\right)$. 


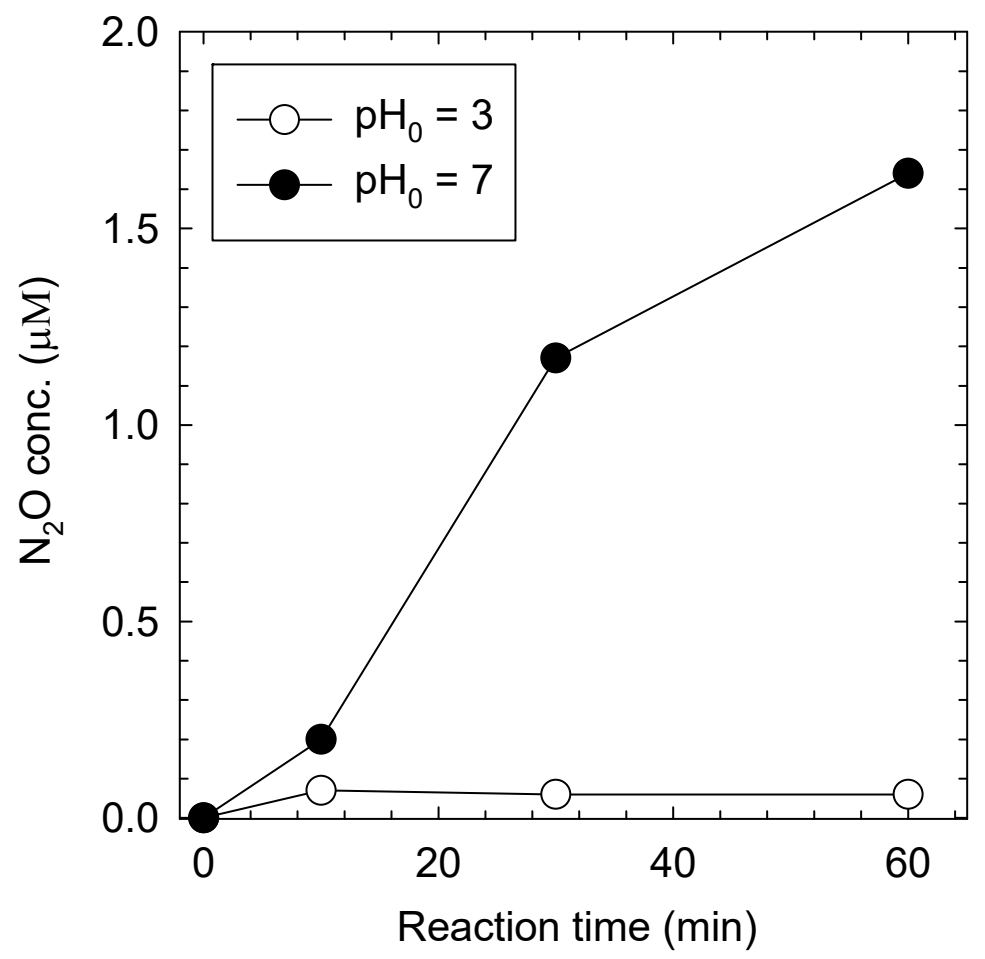

Figure S5. Production of $\mathrm{N}_{2} \mathrm{O}$ in the $\mathrm{Cu}(\mathrm{II}) / \mathrm{HA}$ system at acidic and neutral $\mathrm{pH}$ values $\left([\mathrm{Cu}(\mathrm{II})]_{0}=\right.$ $\left.0.1 \mathrm{mM},[\mathrm{HA}]_{0}=5 \mathrm{mM}\right)$. 


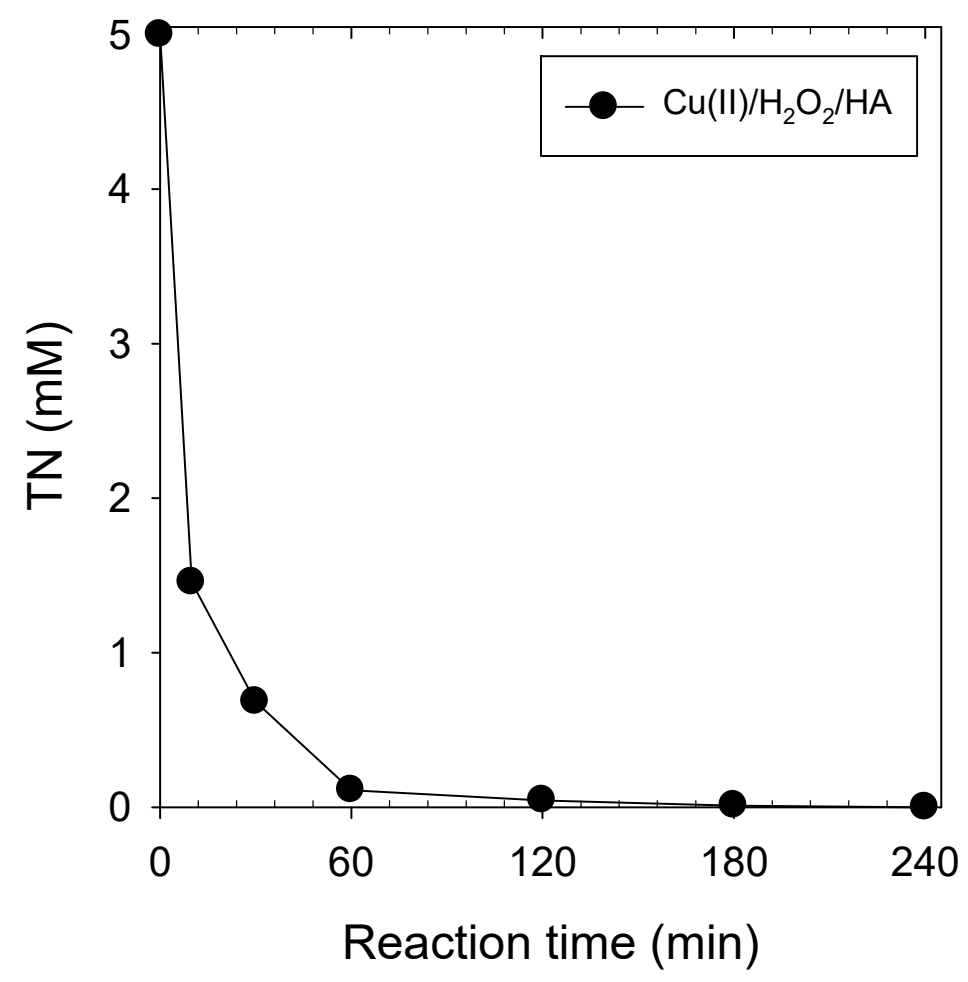

Figure S6. Variation in TN concentration during the decomposition of $\mathrm{HA}$ in the $\mathrm{Cu}(\mathrm{II}) / \mathrm{H}_{2} \mathrm{O}_{2} / \mathrm{HA}$ system $\left([\mathrm{BA}]_{0}=0.1 \mathrm{mM},[\mathrm{Cu}(\mathrm{II})]_{0}=0.1 \mathrm{mM},[\mathrm{HA}]_{0}=5 \mathrm{mM},\left[\mathrm{H}_{2} \mathrm{O}_{2}\right]_{0}=10 \mathrm{mM}, \mathrm{pH}=7\right)$. 


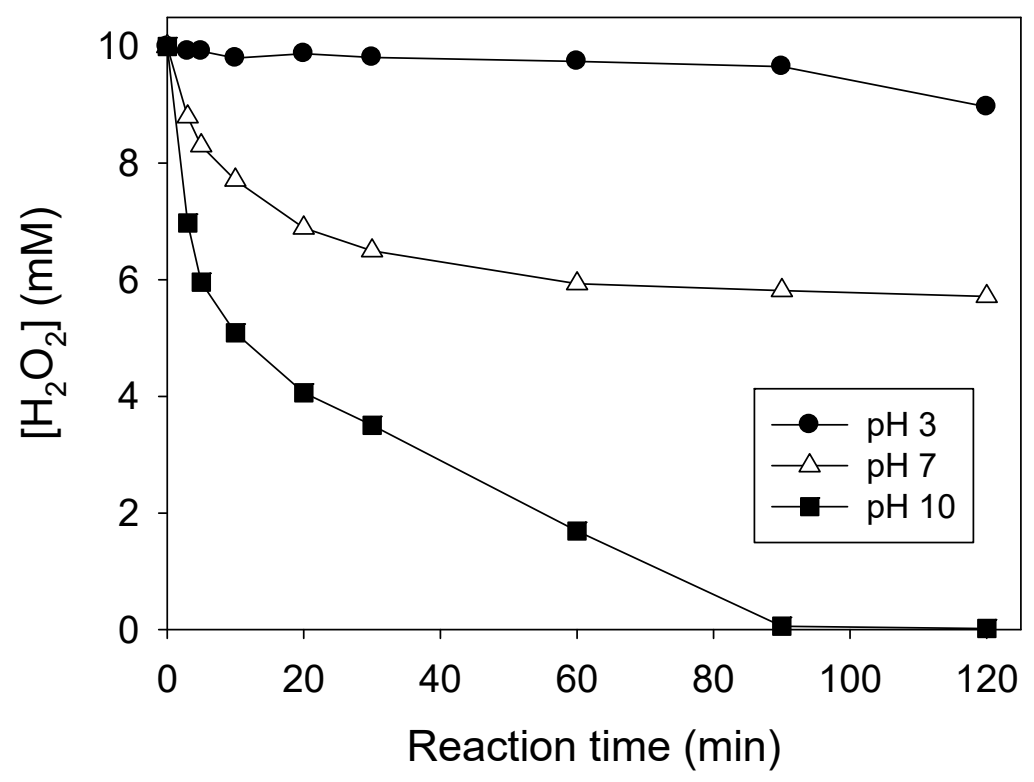

Figure S7. Decomposition of $\mathrm{H}_{2} \mathrm{O}_{2}$ in the $\mathrm{Cu}(\mathrm{II}) / \mathrm{H}_{2} \mathrm{O}_{2} / \mathrm{HA}$ system at different $\mathrm{pH}$ values $\left([\mathrm{BA}]_{0}=\right.$ $\left.0.1 \mathrm{mM},[\mathrm{Cu}(\mathrm{II})]_{0}=0.1 \mathrm{mM},[\mathrm{HA}]_{0}=5 \mathrm{mM},\left[\mathrm{H}_{2} \mathrm{O}_{2}\right]_{0}=10 \mathrm{mM}\right)$. 

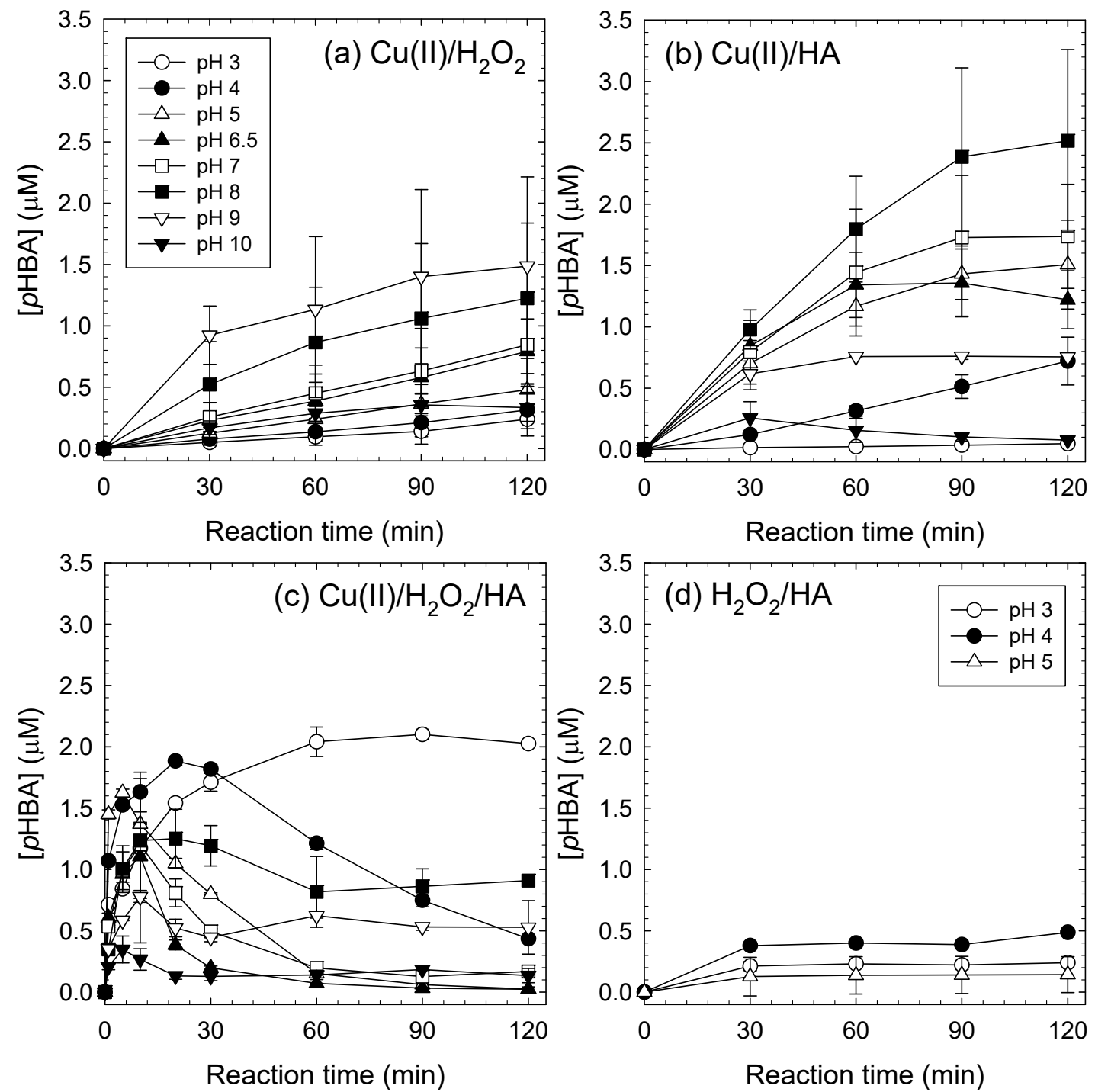

Figure S8. Time-concentration profiles of $p \mathrm{HBA}$ during the BA degradation by (a) $\mathrm{Cu}(\mathrm{II}) / \mathrm{H}_{2} \mathrm{O}_{2}$, (b) $\mathrm{Cu}(\mathrm{II}) / \mathrm{HA}$, (c) $\mathrm{Cu}(\mathrm{II}) / \mathrm{H}_{2} \mathrm{O}_{2} / \mathrm{HA}$, and (d) $\mathrm{H}_{2} \mathrm{O}_{2} / \mathrm{HA}$ systems as different $\mathrm{pH}$ values $\left([\mathrm{BA}]_{0}=0.1 \mathrm{mM}\right.$, $\left.[\mathrm{Cu}(\mathrm{II})]_{0}=0.1 \mathrm{mM},[\mathrm{HA}]_{0}=5 \mathrm{mM},\left[\mathrm{H}_{2} \mathrm{O}_{2}\right]_{0}=10 \mathrm{mM}\right)$ 

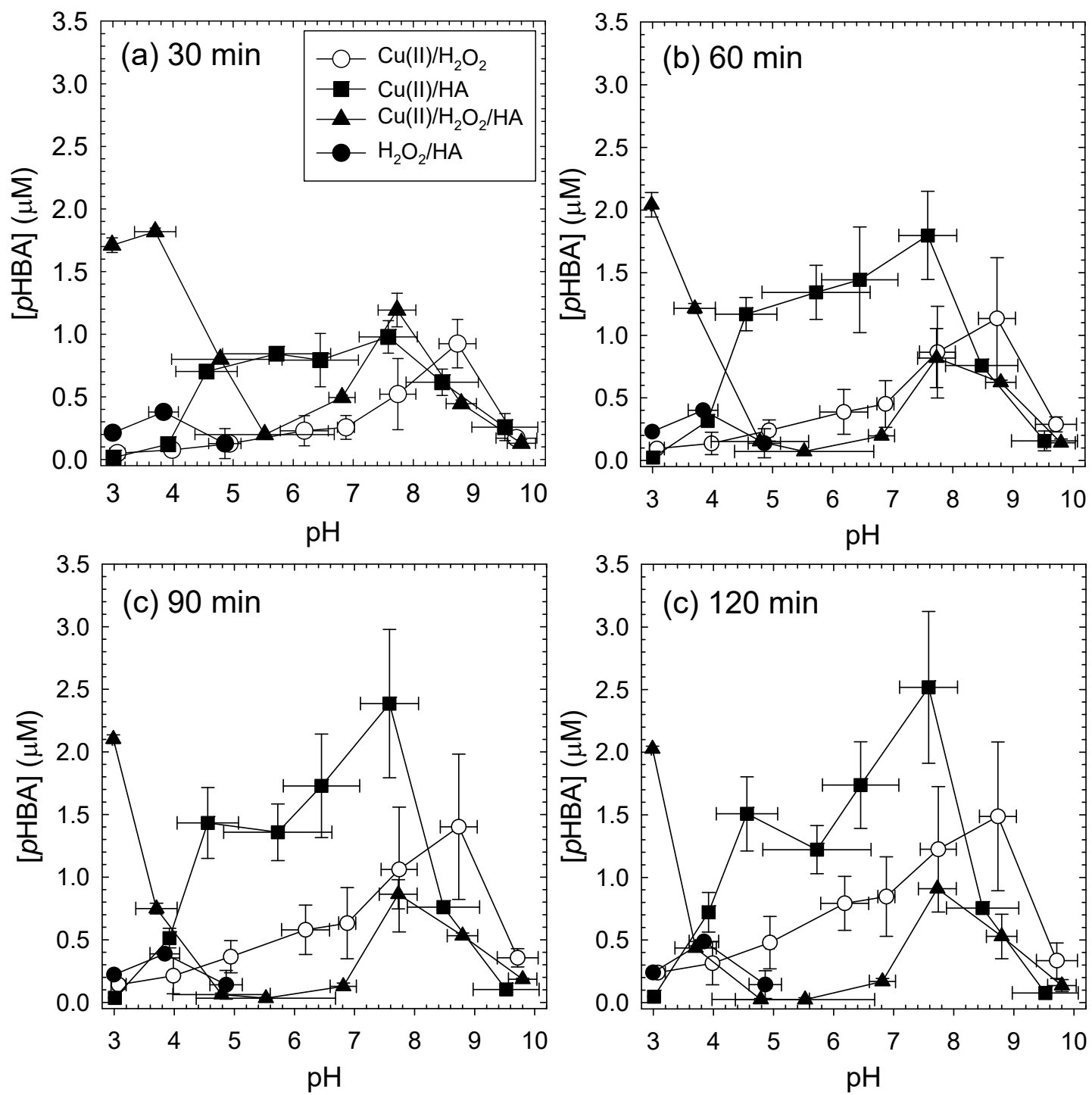

Figure S9. Plots of $\mathrm{pHBA}$ production as a function of $\mathrm{pH}$ at different reaction times (plotted using the data of Figure S2-1) $\left([\mathrm{BA}]_{0}=0.1 \mathrm{mM},[\mathrm{Cu}(\mathrm{II})]_{0}=0.1 \mathrm{mM},[\mathrm{HA}]_{0}=5 \mathrm{mM},\left[\mathrm{H}_{2} \mathrm{O}_{2}\right]_{0}=10 \mathrm{mM}\right)$. 


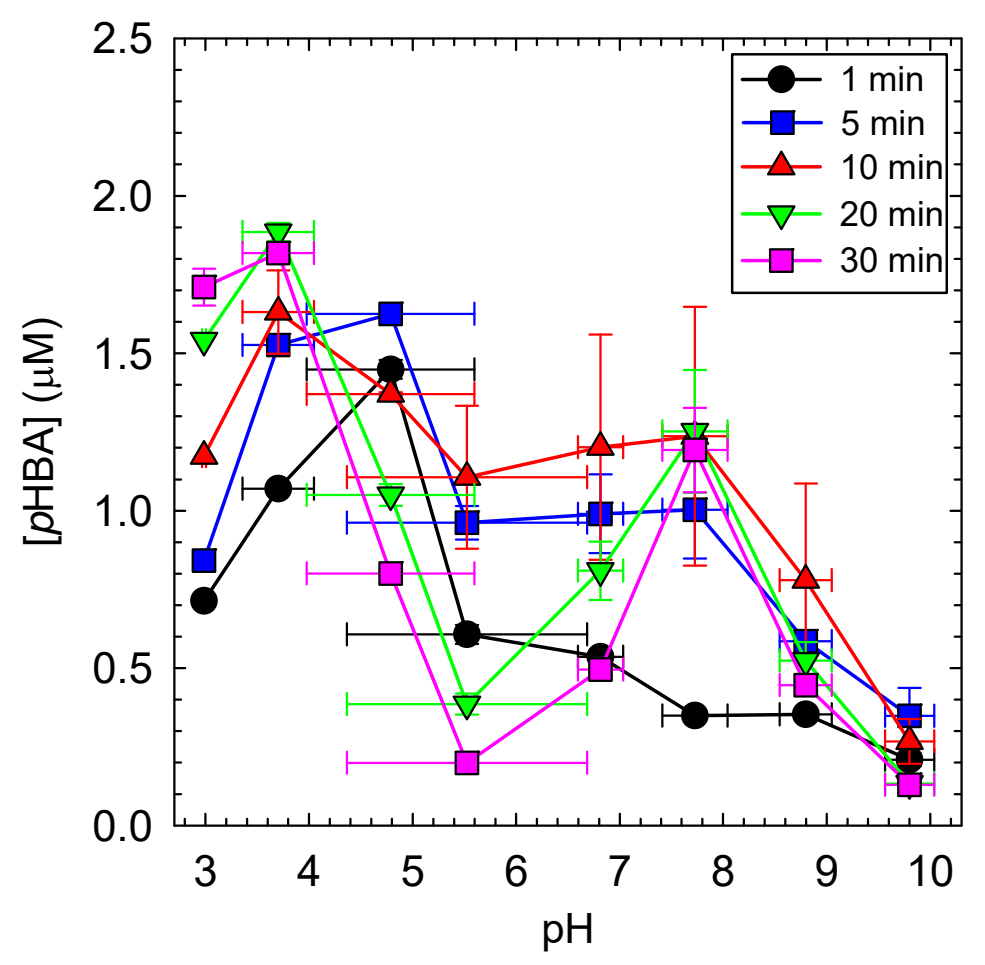

Figure $\mathrm{S} 10$. Production of $p \mathrm{HBA}$ by the $\mathrm{Cu}(\mathrm{II}) / \mathrm{H}_{2} \mathrm{O}_{2} / \mathrm{HA}$ system as a function of $\mathrm{pH}$ in early reaction times (plotted using the data of Figure $\mathrm{S} 8 \mathrm{c})\left([\mathrm{BA}]_{0}=0.1 \mathrm{mM},[\mathrm{Cu}(\mathrm{II})]_{0}=0.1 \mathrm{mM},[\mathrm{HA}]_{0}=\right.$ $\left.5 \mathrm{mM},\left[\mathrm{H}_{2} \mathrm{O}_{2}\right]_{0}=10 \mathrm{mM}\right)$. 


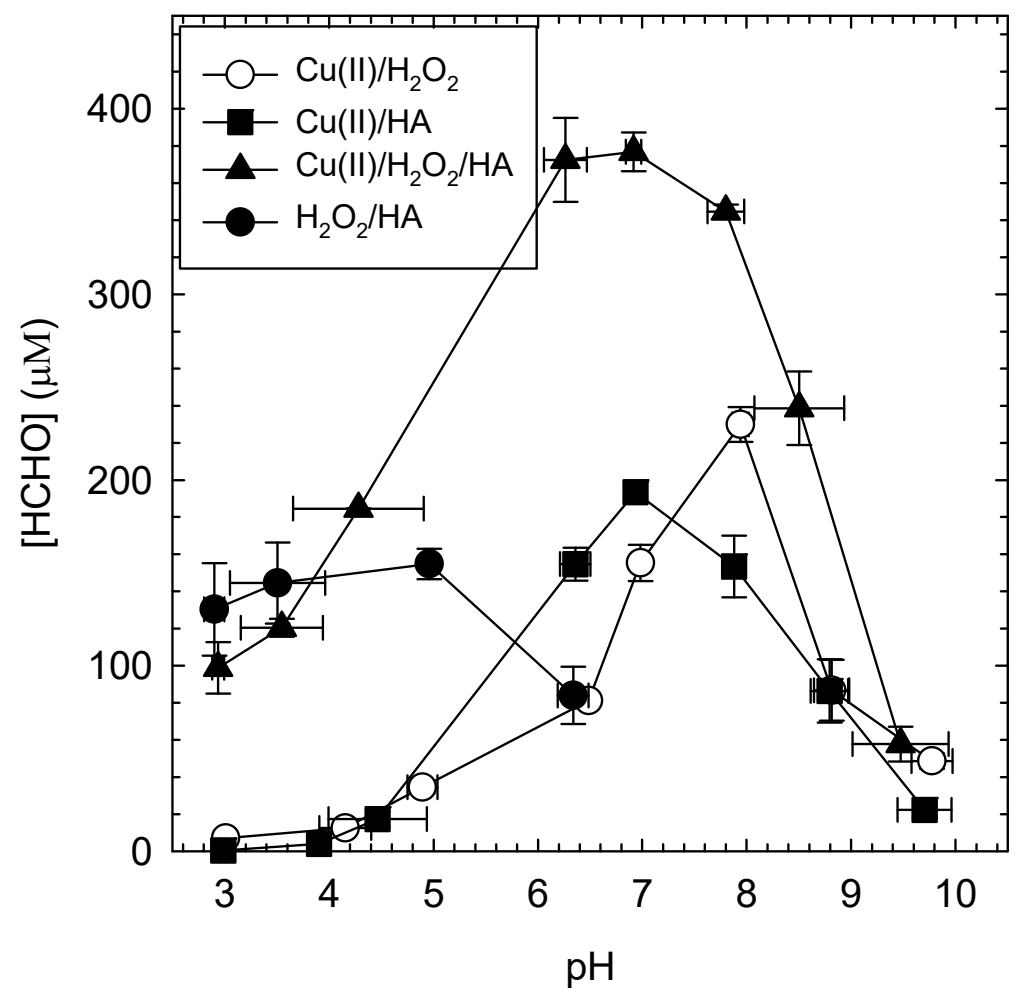

Figure S11. Production of HCHO by different systems as a function of $\mathrm{pH}$. ([Methanol $]_{0}=200 \mathrm{mM}$, $[\mathrm{Cu}(\mathrm{II})]_{0}=0.1 \mathrm{mM},[\mathrm{HA}]_{0}=5 \mathrm{mM},\left[\mathrm{H}_{2} \mathrm{O}_{2}\right]_{0}=10 \mathrm{mM}$, reaction time $\left.=2 \mathrm{~h}\right)$. 


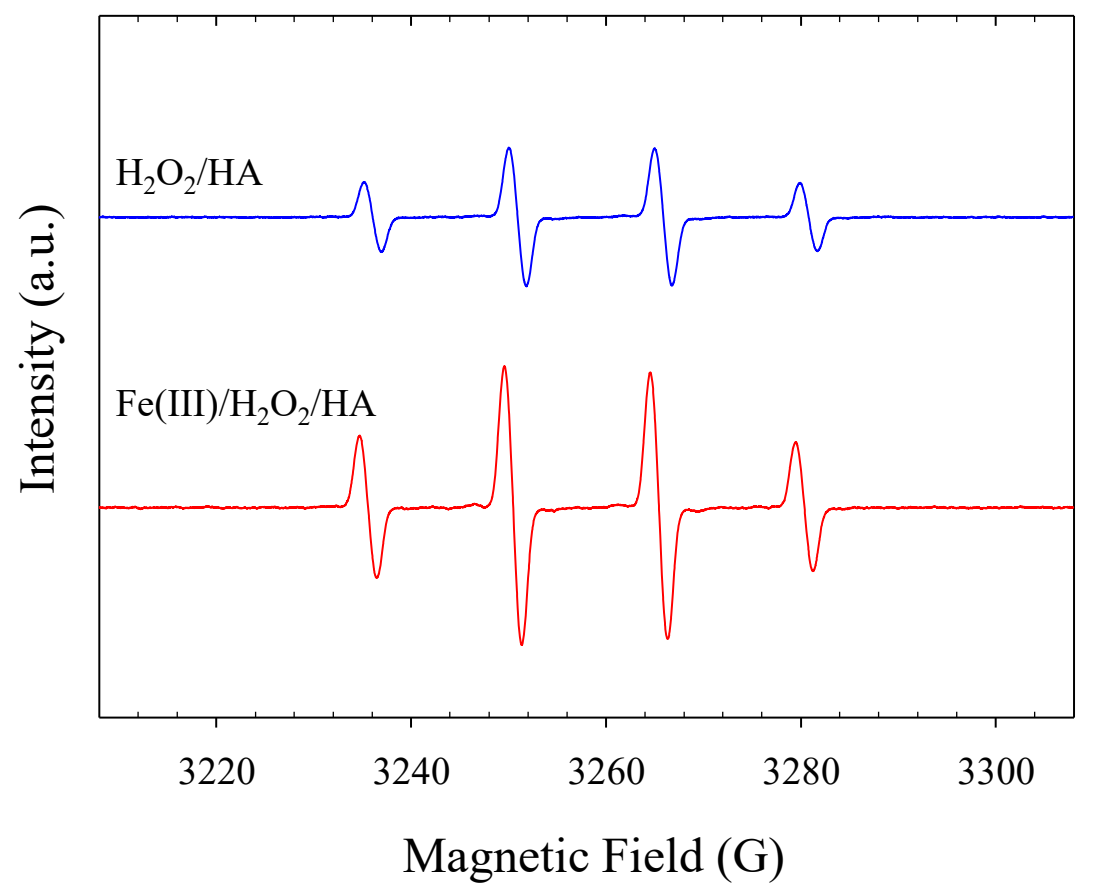

Figure S12. EPR spectra obtained by spin trapping with DMPO in $\mathrm{Fe}(\mathrm{III}) / \mathrm{H}_{2} \mathrm{O}_{2}$ and $\mathrm{Fe}(\mathrm{III}) / \mathrm{H}_{2} \mathrm{O}_{2} / \mathrm{HA}$ systems $\left([\mathrm{DMPO}]_{0}=10 \mathrm{mM},[\mathrm{Fe}(\mathrm{III})]_{0}=0.1 \mathrm{mM},[\mathrm{HA}]_{0}=0.5 \mathrm{mM},\left[\mathrm{H}_{2} \mathrm{O}_{2}\right]_{0}=10\right.$ $\mathrm{mM}, \mathrm{pH}=3$, Reaction time $=10 \mathrm{~min})$. 